\title{
The Stroop effect: It is not the robust phenomenon that you have thought it to be
}

\author{
MIRIAM DISHON-BERKOVITS \\ Bar-Ilan University, Ramat-Gan, Israel \\ and \\ DANIEL ALGOM \\ Tel-Aviv University, Ramat-Aviv, Israel
}

\begin{abstract}
Five experiments demonstrate that context has a powerful effect on the ease with which people can name (Experiments 1-3) or categorize (Experiments 4-5) a stimulus while ignoring another stimulus, irrelevant or conflicting with the target. Selectivity of attention to the target dimension was gauged through Stroop and Garner effects. When the stimulus values along the target dimension and the to-beignored dimension were correlated over the experimental trials, large effects of Stroop and Garner influenced performance. However, when random allocation of values created zero dimensional correlation, the Stroop effects vanished. These results imply that when the nominally irrelevant dimension is in fact correlated with the relevant dimension, participants then attend to the irrelevant dimension and thus open themselves up to Stroop interference. Another variable of context, the relative salience of the constituent dimensions, also affected performance with the more discriminable dimension disrupting selective attention to the less discriminable dimension. The results demonstrate the importance of context in engendering the failure of selective attention and challenge traditional automaticity accounts of the Stroop effect.
\end{abstract}

The Stroop effect (Stroop, 1935) is the demonstration of choice used by instructors of psychology to illustrate the failure of selective attention: Naming the print color of color words is impaired by the meaning of the words, although reading the words is not similarly hindered by irrelevant print color. The tenacity of the asymmetry in interference has earned the phenomenon more than classroom popularity. The Stroop effect has accrued theoretical interest because, presumably, the effect is the inescapable outcome of pitting the automatic process of word reading against the less automatic, or controlled, process of color naming. The mandatory failure of selective attention to color naming ensues. Consequently, the Stroop effect has been considered to be the "gold standard" of attentional processing (MacLeod, 1992). The notions of automaticity (Hasher \& Zacks, 1979; Kahneman \& Treisman, 1984; Logan, 1980; Logan \& Zbrodoff, 1998) and relative speed of processing (e.g., Dunbar \& MacLeod, 1984; Posner, 1978) have indeed served to explain the Stroop phenomenon: Because word reading is more automatic and speedy than color naming, color naming suffers intrusions from involuntary word reading, but reading is not hampered by

This research is based on part of a doctoral dissertation by M.D.-B. in the Department of Psychology, Bar-Ilan University, supervised by D.A. We thank James Pomerantz for his help and insightful comments. M.D.-B. is now at the Department of Psychology, Princeton University. Correspondence concerning this article should be addressed to D. Algom, Department of Psychology, Tel-Aviv University, Ramat-Aviv 69978, Israel (e-mail: algomd@freud.tau.ac.il). conflicting print color. More than 60 years after its discovery, the Stroop phenomenon continues to fascinate investigators and has sustained a substantial amount of research (see MacLeod, 1991, for a comprehensive review).

In the present study, we challenge both the alleged robustness of the Stroop phenomenon and its traditional explanations. We demonstrate that the effect is malleable experimentally by several heretofore neglected stimulus factors. By judiciously manipulating these factors, one is able to determine the direction, the magnitude, and in fact, the very appearance of the Stroop effect. Hence, the effect is neither robust nor, indeed, inevitable. These results, in turn, cast doubt on theories positing the automatic activation of the meaning of words.

Chief among the recently discovered factors are the relative discriminability of the word and color dimensions and the correlation between these constituent dimensions of the Stroop stimulus. Discriminability relates to the psychological difference separating stimulus values along the color and the word dimensions (e.g., Garner \& Felfoldy, 1970; Melara \& Mounts, 1993). Discriminability is matched when the print colors used are as easily distinguishable from one another as are the color words. Notably, the discriminability of values along the constituent dimensions has been neither appreciated nor measured in earlier studies of the Stroop phenomenon. When it was, Melara, Algom, and their associates (Algom, Dekel, \& Pansky, 1993, 1996; Arieh \& Algom, 1997; Melara \& Algom, 1996; Melara \& Mounts, 1993, 1994; Pansky \& Algom, 1999; Sabri, Melara, \& Algom, in press; Shalev 
A.
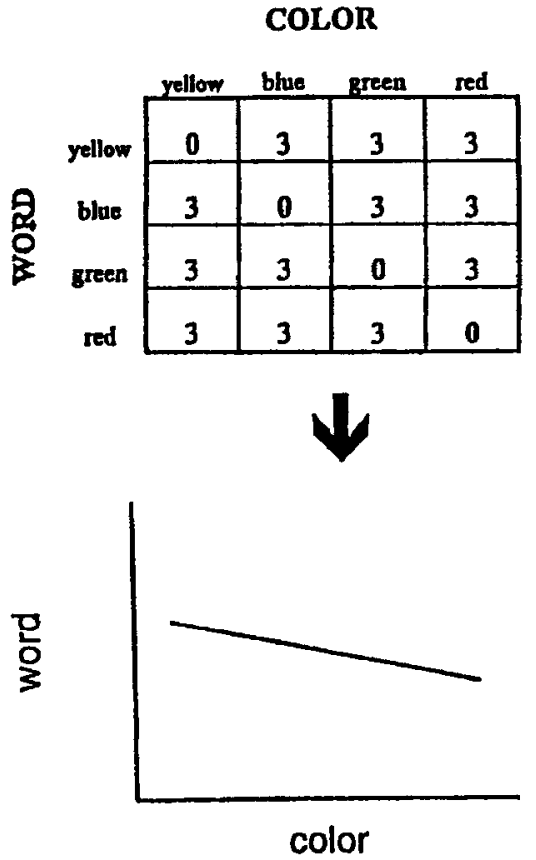

B.

\begin{tabular}{|c|c|c|c|c|}
\hline & & $\mathrm{CO}$ & & \\
\hline & yellow & blue & green & red \\
\hline yellow & 9 & 3 & 3 & 3 \\
\hline blue & 3 & 9 & 3 & 3 \\
\hline green & 3 & 3 & 9 & 3 \\
\hline re & 3 & 3 & 3 & 9 \\
\hline
\end{tabular}

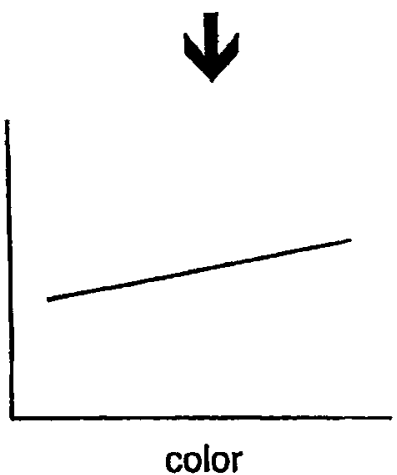

C.

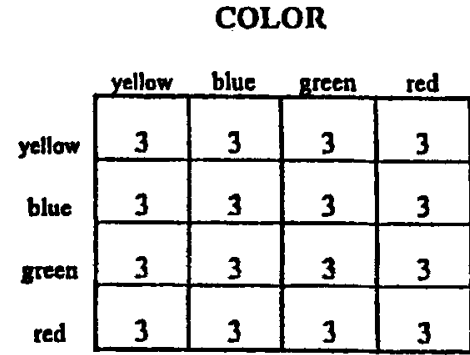

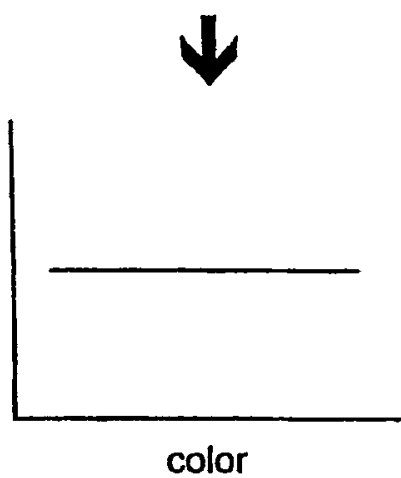

Figure 1. Allocation of words and colors to form the set of color-word stimuli in three experimental situations. Panel $A$ depicts a negative correlation, in which only incongruent stimuli are included in the set. The negative slope of the regression line illustrates the fact that one dimension is predictive of the other. Panel B depicts a positive correlation, in which the conditional probability of a color (word) given a word (color) is greatest for the congruent words (colors). The resulting predictive relationship is illustrated by the positive slope of the regression line. Panel $\mathrm{C}$ depicts strictly random allocation (zero correlation), in which the conditional probability of a color (word) given a word (color) is the same for all colors (words). Under this policy, word is not predictive of color (and vice versa), a fact demonstrated by the zero slope of the regression line.

\& Algom, 2000) have reproduced the usual Stroop asymmetry for words that were more discriminable than colors. Strikingly, when the words and the colors were matched in discriminability, the asymmetry disappeared, and when the colors were more discriminable than the words, the asymmetry actually reversed. Hence, the magnitude, the direction, and even the presence of the Stroop effect could be predictably fabricated by modulating relative dimensional discriminability. Another major factor of context, dimensional correlation, specifies the rule by which the colors and the words are combined to form the Stroop stimuli; hence, dimensional correlation is coextensive with the experimental design.

\section{The Anatomy of the Stroop Experiment}

Figure 1 portrays three possible ways of allocating values of color and word to form the stimuli in any Stroop experiment. In panel A, the diagonal of the matrix is empty: None of the words names its print color (i.e., none of the stimuli is congruent). This was the arrangement employed by Stroop (1935) in his classic study. In Stroop's study, the words conflicted with the colors on all of the experimental trials. Stroop thus established a negative correlation between the word and the color dimensions in his study. Modern studies of the Stroop effect do include congruent as well as incongruent stimuli in the experimen- tal set-usually, in equal numbers. Espousing this design, however, one establishes a positive correlation between the Stroop dimensions: The conditional probability of a word (color), given its matching color (word), is greater than that of any other word (color). Only in panel $\mathrm{C}$ do we depict a truly random allocation of words and colors to form the experimental stimuli. This is the one stimulus design in which there is a zero correlation between dimensions. The conditional probability of a word (color), given a color (word), is the same for all words (colors).

Dimensional correlation is fatal for selective attention. If there is a correlation between color and word built into the experimental design, then one dimensional value of the Stroop stimulus carries information about the other. Garner (1962) cautioned that "subjects will find any cue at all to which they can relate their responses, and if a small artifactual cue exists which is, strictly speaking, irrelevant to the defined task, the subject will nevertheless relate his responses to that ... cue" (p. 341). An irrelevant dimension that is correlated with the target dimension provides precisely such a cue. Presented with a Stroop stimulus, the participant has a better-than-chance probability of identifying the word once he or she has noticed the color. Similarly, noticing the word provides the participant with information about the color. Humans are notorious at capturing covariation or information lurk- 
ing in their environment (e.g., Alloy \& Tabachnik, 1984; Bar-Hillel \& Wagenaar, 1991; Kareev, 1995; Kareev, Lieberman, \& Lev, 1997): By establishing a(ny) correlation over trials between color and word, the Stroop experimenter virtually dictates the diversion of attention toward the irrelevant dimension. Because they attend to the nominally irrelevant dimension (to maximize performance), the participants open themselves up to Stroop interference. To evaluate the dependence of the Stroop effect on the correlation between the stimulus dimensions, we applied Garner's (1974) speeded classification paradigm.

\section{Garner's Speeded Classification Paradigm}

The participant is asked to classify the Stroop stimulus on the relevant dimension-color or word-and ignore variation on the irrelevant dimension. Performance is assessed across the following four conditions. In the filtering condition, the participant is asked to classify values on one dimension - say, whether the color is blue or redwhile ignoring irrelevant variation on the second dimension of word. In the baseline condition, the participant is again asked to classify values on the criterial dimension (whether the color is blue or red), but the values on the irrelevant dimension are held constant (e.g., the word is always red). In the correlated dimensions conditions, the participant is asked yet again to classify values on the criterial dimension. However, values on the irrelevant dimension vary in a perfectly correlated manner throughout the sequence. In the positively correlated dimensions condition, word and color always match (e.g., the word red always appears in red); in the negatively correlated dimensions condition, word and color always mismatch (the word red always appears in blue). The original Garner paradigm thus entails conditions with zero (filtering) and perfect (the positively and negatively correlated dimensions tasks) contingency and binary-valued dimensions. In the present study, we enhanced ecological validity by using smaller-than-unity coefficients of dimensional correlation and many-valued Stroop dimensions.

The ability to attend selectively is measured by comparing performance in the baseline condition, in which the irrelevant dimension is held constant, with performance in the filtering condition, in which the two dimensions are varied orthogonally. If performance at baseline equals that in filtering, selective attention is perfect. The parity shows that the participants were able to focus on one dimension (color) without suffering distraction from irrelevant variation on another dimension (word). Conversely, if filtering performance is worse than baseline, selective attention has failed. The difference in performance between the filtering and the baseline conditions is called Garner interference (Pomerantz, 1986), and it reflects an inability to focus exclusively on the relevant dimension. Pairs of dimensions that give rise to Garner interference are called integral or interacting dimensions.
Pairs of dimensions that do not produce significant Garner interference are called separable dimensions.

\section{Deriving Stroop and Garner Interference}

Stroop interference and Garner interference are separate measures of (the failure of) selective attention. The Stroop effect is assessed by calculating the difference in performance between trials in which the stimulus dimensions correspond (congruent trials) and trials in which the dimensions conflict (incongruent trials). Three measures satisfy this definition in the full Garnerian regime. In the filtering condition, the relevant and the irrelevant dimensions vary orthogonally. Therefore, on half the trials the color and the word dimensions correspond, and on half they conflict. The difference in performance between the two types of trials yields one measure of Stroop congruity. The same Stroop calculation applies to the baseline condition. A third measure of the Stroop effect derives from the difference in performance between the positively correlated dimensions condition (all the stimuli correspond) and the negatively correlated dimensions condition (all the stimuli conflict). This last index is a between-conditions measure (as is Garner interference), whereas the former two are within-condition measures. Conceptually, Garner interference registers the fact that the observer noticed irrelevant variation. Stroop interference registers the fact that the observer noticed the content of the irrelevant dimension. Because one cannot notice the content of irrelevant variation without also noticing the variation, Stroop interference implies Garner interference, although the reverse is not true (Pomerantz, 1983, 1986; Pomerantz, Pristach, \& Carson, 1989).

\section{The Present Study}

In five experiments, we manipulated the magnitude of the correlation between the constituent dimensions from positive to zero to negative. In the first three experiments the correlation was varied, with the dimensions roughly matched in discriminability, whereas in the last two experiments the correlation was varied, with the dimensions mismatched in discriminability. To overcome the limitations posed by the small set of available colors (and color words), in Experiments 1-3 we used the spatially separated (Kahneman \& Chajczyk, 1983; MacLeod \& Hodder, 1998) word-word (Glaser \& Glaser, 1989; MacLeod \& Hodder, 1998) version of the Stroop task, with city and country as the Stroop dimensions. In Experiments 4-5, we used words embedded within pictures as stimuli and categorization, rather than naming, for responding. If correlation (and discriminability) fail to affect the Stroop phenomenon, the traditional approach is vindicated. If, on the other hand, correlation does determine the outcome, engendering the appearance of the Stroop effect or its evaporation, the results are incompatible with an automaticity account of this central phenomenon of cognitive psychology. 
Table 1

Percentage of Error in the Different Conditions of Experiments 1-5

\begin{tabular}{cccccc}
\hline & \multicolumn{5}{c}{ Condition } \\
\cline { 2 - 6 } Experiment & $R$ & $C+$ & $C-$ & $B$ & Mean \\
\hline 1 & 4.1 & 1.5 & 2.9 & 0.8 & 2.3 \\
2 & 2.2 & 3.8 & 3.5 & 2.8 & 3.1 \\
3 & 1.7 & 2.5 & 5.0 & 2.6 & 3.0 \\
4 & 3.0 & 2.1 & 3.6 & 5.7 & 3.6 \\
5 & 4.1 & 4.0 & 5.5 & 3.2 & 4.1 \\
\hline
\end{tabular}

\section{EXPERIMENT 1}

\section{Method}

Participants. The participants were 17 Bar-Ilan University undergraduates, who took part in the experiment in partial fulfillment of course requirements.

Stimuli and Apparatus. We used seven country names (Austria, Belgium, Brazil, China, England, India, and Israel) and the respective names of 7 major cities in these countries (Salzburg, Antwerp, Rio de Janeiro, Shanghai, Oxford, Bombay, and Netanya) to create a set of 49 country-city compounds. A congruent combination consisted of the name of a city appearing along with the name of its home country, whereas an incongruent combination consisted of the name of a city appearing along with the name of a foreign country. The position-above and below the center of the computer screen - of the names of the country and city was alternated randomly from trial to trial. The geographical items used were determined on the basis of pilot testing. A pilot study conducted on an independent sample of 118 students indicated that of 43 cities in as many countries tested, the seven countries and 7 cities selected were ranked the most familiar (all the respondents in the sample judged correctly the respective geographical relations).

The stimuli were generated in Microsoft Word (Hebrew font Miriam, size 24) by an IBM-compatible (PC-486) microcomputer and displayed on a super-VGA 14 -in. color monitor. On a trial, a pair of words appeared (one denoting a country, another denoting a city) above and below the screen center. The stimuli appeared black over a light gray background at around the center of the screen. Each word was centered horizontally and shifted vertically by $1 \mathrm{~cm}$ above or below the center of the screen. To avoid adaptation, we introduced a trial-to-trial spatial uncertainty of up to 10 pixels around the target locations. Viewed from a distance of approximately $60 \mathrm{~cm}$, single words subtended $0.57^{\circ}$ of visual angle in height and between $1.33^{\circ}$ and $5.16^{\circ}$ of visual angle in length.

From the 49 country-city pairs, we constructed four experimental tasks. Our goal was to manipulate the degree of dimensional correlation. To effect the changes in correlation, in each task we introduced a different value for the probability of a city $B_{m}$, given a country $A_{n}$. We varied the conditional probability $p\left(B_{m} \mid A_{n}\right)$ across the four experimental tasks. A brief description of each task follows

In the $R$ (random) task, we allocated the dimensional values in a truly random fashion. We instructed our computer program (specifically designed for the study) to use independent random sampling so that, on a trial, each of the 49 country-city combinations had exactly the same probability of being sampled and presented for view. The conditional probability of a country (city), given a city (country), was the same for all the countries (cities). Therefore, given the 7 (country) $\times 7$ (city) matrix, each country-city combination had a probability of .02 of appearing.

In the $C+$ (positive correlation) task, we set the probability for congruent country-city combinations higher than that for incongruent country-city combinations. Specifically, we set the probability for the former to be 6.5 times higher than it would have been expected to be on the basis of strictly random sampling. As a result, $93 \%$ of the country-city combinations sampled were congruent, and $7 \%$ were incongruent.

In the $C-$ (negative correlation) task, we set the probability for incongruent country-city combinations higher than that for congruent country-city combinations. Again, our point of departure for establishing the negative correlation over trials was that of a strictly random sampling of the city-country pairs. We set the probability for congruent country-city combinations to be four times smaller than that expected on the basis of random sampling. Consequently, $3.5 \%$ of the country-city combinations were congruent, and $96.5 \%$ were incongruent.

Finally, in the $B$ (baseline) task, we varied the names of the countries randomly, whereas the city name was held constant throughout the task (a different invariant city for each participant). Therefore, in the baseline task, we only varied the relevant (and, hence, the to-be-named) dimension, whereas the irrelevant dimension was held at a constant value.

The various tasks used are easily summarized in terms of the correlation established between the values of the city and country dimensions. In two tasks $\left(C^{+}, C^{-}\right)$, the names from the two dimensions covaried from trial to trial in a systematic fashion, once positively and another time negatively. In the $R$ task, values from the two dimensions covaried randomly, creating a zero correlation between city and country. In the $B$ task, a zero correlation was created between country and city by holding the irrelevant dimension of city at a constant value.

Each task consisted of 49 trials. From the $7 \times 7$ matrix, we sampled 49 country-city pairs randomly, subject to the special constraints of each task. Because we used sampling with replacement, a given pair could appear several times during a given task or not at all.

Procedure. The participants were tested individually in a dimly lit room. Each participant performed in each of the four tasks, with task order varying in a random fashion from participant to participant. On a trial, a pair of names vertically flanking the center of the screen appeared, one of a country and another of a city. We introduced positional uncertainty so that there was trial-to-trial variation in the relative position of the country and the city. The participants were instructed to name the relevant dimension, country, and to ignore the irrelevant dimension, city. They were encouraged to respond quickly without making errors. Responses were made orally and recorded by a microphone. Each stimulus remained on display for $500 \mathrm{msec}$ or was response terminated, whichever came first. We measured the time elapsed between the onset of stimulus presentation and the vocal response in milliseconds. The experimenter recorded the correctness of the response.

The first nine trials of each task consisted of practice trials (unbeknownst to the participant) preserving the same correlational structure as that created in the ensuing experimental task. Each task was followed by a short rest. The participant initiated instructions to the next task by pressing the left key of the mouse. The experiment lasted approximately $15 \mathrm{~min}$.

Data analysis. All the analyses were performed using the original set of response time (RT) distributions (reflected in large values of the degrees of freedom parameter). Trials in which the participant's mean RT was below $400 \mathrm{msec}$ or exceeded $1,800 \mathrm{msec}$ were excluded from the analysis. Planned comparisons among pairs of conditions were performed using the Duncan test with a significance level of .05 . The error rates were very low throughout the experimental conditions (see Table 1), as were the correlations between error and RT (coefficients of .08, .11, .16, .06, and .06, respectively, in Experiments 1-5)

\section{Results}

Shown in Figure 2 (panel A, shaded columns) are the average naming latencies for country obtained in the four 


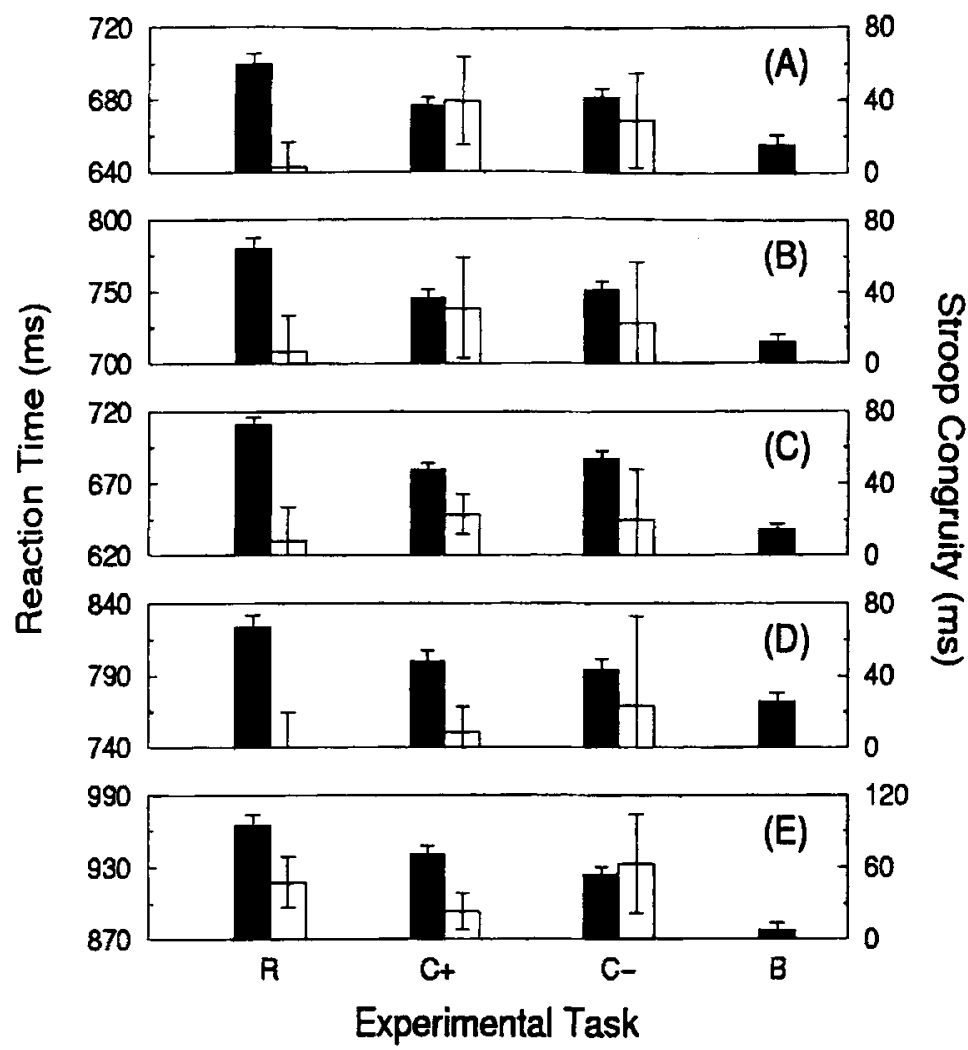

Figure 2. The shaded columns depict Garner effects: Average response times (with standard error bars) to name or categorize the target dimension in four experimental conditions - $R$ (random allocation of dimensional values to create the experimental stimuli), $C+$ (positively correlated dimensions), $C-$ (negatively correlated dimensions), and $B$ (the irrelevant dimension is held constant). Garner interference is the difference between the $R$ and the $B$ tasks. The empty columns depict Stroop effects: Average magnitudes of congruity (with standard error bars) in the $R, C+$, and $C$ - conditions. Each column represents the difference in performance between congruent and incongruent stimuli within a condition. In panels $A$ to $E$, we depict, respectively, the results of Experiments 1-5.

experimental tasks. The mean RTs to name the 49 countries differed across the four tasks in a systematic way. The best performance, as Figure 2 shows, was obtained in the baseline task, in which the name of the irrelevant city was held constant. When the participants had to name countries with the name of the to-be-ignored city invariant across trials, the mean RT was $655.3 \mathrm{msec}$. By contrast, when irrelevant city varied from trial to trial in a random fashion, performance deteriorated to $699 \mathrm{msec}$. The 43.7msec difference in performance- Garner interferencewas highly significant $\left[t(1439)=5.89, M S_{\mathrm{e}}=7.42, p<\right.$ $.001]$. The appreciable amount of Garner interference obtained signals the failure of selective attention to the target dimension of country: Our participants could not ignore variation along irrelevant city when naming the relevant country.

Figure 2 also shows that naming country in the correlated dimensions tasks was faster than naming country in the random task. Mean RTs in the $C+$ and $C$ - tasks were
676.5 and $681.8 \mathrm{msec}$, respectively; the $5.3-\mathrm{msec}$ difference was not significant statistically $[t(1447)=-0.76$, $\left.M S_{\mathrm{e}}=6.99, p>.2\right]$. The participants named countries in the $C+$ task $22.5 \mathrm{msec}$ faster, on average, than in the $R$ task $\left[t(1473)=3.13, M S_{\mathrm{e}}=7.19, p<.01\right]$, and, similarly, participants named countries in the $C$ - task $17.2 \mathrm{msec}$ faster, on average, than in the $R$ task $[t(1436)=2.28$, $\left.M S_{\mathrm{e}}=7.55, p<.05\right]$. Therefore, when the irrelevant dimension covaried with the criterial dimension, whether positively or negatively, the participants reaped performance gains. The superior performance in the correlated dimensions tasks, as compared with that obtained in the random task, underscores the importance of dimensional correlation. The participants captured the built-in correlation between dimensions (even when the correlation was negative) to improve their performance, as compared with that in a condition entailing zero dimensional correlation.

However, Figure 2 also reveals that the participants did not perform better in the correlated dimensions tasks 
than in the baseline task. Instead, performance in the $B$ task was faster by $21.2 \mathrm{msec}$, as compared with that in the $C+\operatorname{task}\left[t(1450)=3.09, M S_{\mathrm{e}}=6.85, p<.01\right]$, and by $26.5 \mathrm{msec}$, as compared with performance in the $C$ - task $\left[t(1413)=3.67, M S_{\mathrm{e}}=7.22, p<.001\right]$. The baseline condition was the easiest one to perform, owing perhaps to the spatial separation of city and country in this experiment (unlike the original Stroop task, in which the two dimensions are integrated onto the same stimulus). An overall analysis of variance (ANOVA) performed on the entire data of Experiment 1 confirmed the reliability of the differences in performance observed across the $R, C+, C-$, and $B$ tasks $\left[F(3,2886)=12.44, M S_{\mathrm{e}}=2.56, p<.001\right]$.

Our second measure of selective attention, Stroop congruity, was defined by the difference in RT between trials in which the dimensions corresponded (a city appearing along with its home country, congruent trials) and trials in which the dimensions conflicted (a city appearing along with the name of a foreign county, incongruent trials). Because each of our tasks entailed both congruent and incongruent combinations (we did not use perfect correlations), Stroop congruity could be calculated in each. Note that, although possible to calculate, the interpretation of Stroop congruity in the $B$ task is moot, given the fact that the irrelevant dimension is held constant (i.e., the task actually is unidimensional). Calculated values of Stroop congruity in the $B$ tasks amounted to $2,1.8,-38.2$,

-10.5 , and $-2.4 \mathrm{msec}$, respectively, in Experiments 1-5, with only the large negative value in Experiment 3 significant statistically.

The results with respect to Stroop congruity are also shown in panel A of Figure 2 (empty columns). Again, the results differ across tasks in a predictable fashion. Consider first the Stroop congruity obtained in the $C+$ and $C-$ tasks. In the $C+$ task, the participants named countries appearing along with domestic cities in an average of $675 \mathrm{msec}$ but named countries appearing along with foreign cities in an average of $715 \mathrm{msec}$. The $40-\mathrm{msec}$ difference between the congruent and the incongruent trials defined a significant effect of Stroop congruity $\left[t(741)=1.59, M S_{\mathrm{e}}=25.05, p=.05\right]$. Similarly, in the $C-$ task, the mean RTs were 654 and $683 \mathrm{msec}$, respectively, for congruent and incongruent trials, amounting to a Stroop effect of $29 \mathrm{msec}\left[t(704)=1.11, M S_{\mathrm{e}}=26.22\right.$, $p=.13$ ]. Of the 17 participants, 14 had at least a modicum of a Stroop effect in the $C$ - task (i.e., for these persons, the difference in RTs between the incongruent and the congruent stimuli was greater than zero). Chance probability for this pattern falls well below $1 \%$. Hence, not only did irrelevant variation take a toll on performance (Garner interference), but the participants also took notice of the content of the irrelevant dimension to speed up or slow down performance (Stroop congruity).

Finally, consider the Stroop effect in the $R$ task. In the random task, in which there was a zero correlation between country and city, Stroop congruity was wholly eliminated. The mean RTs were 699.4 and $696.7 \mathrm{msec}$ for congruent and incongruent trials, respectively; the mi- nuscule difference of $2.6 \mathrm{msec}$ was insignificant $[t(730)=$ $\left.0.17, M S_{\mathrm{e}}=15.71, p>.4\right]$. The absence of a Stroop effect in the $R$ task, numerically as well as statistically, is striking. When one stimulus dimension carries no information about another dimension, selective attention to either dimension is perfect: None intrudes on performance with the other, and as a result, no Stroop effect ensues.

\section{Discussion}

The substantial amount of Garner interference obtained means that our participants did not attend selectively to country and ignore irrelevant variation in city: Country and city interacted in perception. The presence of Stroop congruity in the $C$ tasks implies that the participants were sensitive to the content of the variation along the irrelevant dimension. Because the participants noticed the momentary value of irrelevant city, corresponding or conflicting, they suffered Stroop interference in the context of the positively and negatively correlated conditions. These results lend support to the dictum laid down in the introduction: Dimensional correlation and dimensional selectivity of attention are incompatible.

The elimination of Stroop congruity in the $R$ task is the signature of the present experiment. When dimensional values were allocated in a strictly random fashion, Stroop congruity vanished completely. These findings are incompatible with an automaticity account of the Stroop phenomenon. On the basis of the latter approach, one would predict a modicum of Stroop effect to emerge under all conditions. The conspicuous failure of a Stroop effect to emerge under zero dimensional correlation supports instead our contextual approach. The Stroop phenomenon is an optional effect, malleable by variables of context.

Given the theoretical burden of these results, we deemed a full replication of the experiment warranted. In Experiment 2 , city was the target dimension.

\section{EXPERIMENT 2}

\section{Method}

Participants. The participants were 15 Bar-Ilan University undergraduates, none of whom had performed in Experiment 1 .

Stimuli and Apparatus. The stimuli and apparatus were the same as those of Experiment 1.

Procedure. The procedure was that of Experiment 1. As in Experiment 1 , on a trial, a pair of names appeared, one of a country and another of a city. However, in Experiment 2, city was the relevant dimension. The participants were encouraged to name the city (and ignore the country) as quickly as possible without making errors.

\section{Results}

In panel B of Figure 2 (shaded columns), we present the mean RTs needed to name the 49 cities in the four experimental tasks. Again, the best and worst performances ( $714.3 \mathrm{vs} .780 .5 \mathrm{msec}$ ) were obtained in the baseline and random tasks, respectively. The difference, $66.2 \mathrm{msec}$, amounted to a Garner interference that was highly significant $\left[t(1359)=7.24, M S_{\mathfrak{e}}=9.14, p<.001\right]$. These re- 
sults duplicate the findings of Experiment 1: The participants could not ignore variation along the irrelevant dimension while naming the relevant dimension.

Performance in the correlated dimensions tasks was better than that in the random task. Average naming times in the $C+$ and $C$ - tasks were 745.3 and $750.9 \mathrm{msec}$, respectively; the 5.6-msec difference was not significant statistically $\left[t(1367)=-0.63, M S_{\mathrm{e}}=9.02, p>.2\right]$. Mean naming time in the $C+$ task was faster by $35.3 \mathrm{msec}$ than that in the $R$ task $\left[t(1368)=3.81, M S_{\mathrm{e}}=9.25, p<.001\right]$. Likewise, mean naming time in the $C-$ task was faster by $29.6 \mathrm{msec}$ than that in the $R$ task $\left[t(1365)=3.05, M S_{\mathrm{e}}=\right.$ $9.71, p<.01$ ] . As in Experiment 1, the participants captured the built-in correlation between the dimensions (whether positive or negative) to enhance their performance, as compared with that in the random task.

Performance in the $B$ task was again superior to those in the $C$ tasks. The participants named cities in the $B$ task $31 \mathrm{msec}$ faster, on average, than in the $C+$ task $[t(1361)=$ $\left.3.68, M S_{\mathrm{e}}=8.40, p<.001\right]$ and $36.6 \mathrm{msec}$ faster, on average, than in the $C-\operatorname{task}\left[t(1358)=4.11, M S_{\mathrm{e}}=8.91\right.$, $p<.001]$. Again, we implicate the spatial separation of the city and the country components as the source of these differences. An overall ANOVA confirmed the reliability of the differences in performance obtained across the four tasks $\left[F(3,2726)=17.78, M S_{\mathrm{e}}=3.24, p<.001\right]$.

In panel $B$ of Figure 2, we also provide the results on Stroop congruity (empty columns). In the $C+$ task, mean naming times for congruent and incongruent pairs of country and city were 743.8 and $774.7 \mathrm{msec}$, respectively, amounting to a Stroop congruity of $30.8 \mathrm{msec}[t(684)=$ $\left.1.08, M S_{\mathrm{e}}=28.62, p=.14\right]$. Of the 15 participants, only 3 failed to show a Stroop effect in the $C+$ task. The chance probability for this result is .01 . In the $C-$ task, mean RTs for congruent and incongruent pairs were 729.5 and $751.8 \mathrm{msec}$, respectively, producing a Stroop effect of $22.3 \mathrm{msec}\left[t(681)=0.65, M S_{\mathrm{e}}=34.48, p=.25\right]$. In this task, too, only 4 participants failed to evince the dominant pattern of a Stroop effect -an unlikely event (with a probability of less than $1 \%$ ) under the hypothesis of equal performance across stimulus type. The Stroop effects obtained in the correlated dimensions tasks, although large, were somewhat less reliable statistically than we had expected. Nevertheless, they constituted the dominant pattern of data. People do notice the value of the irrelevant dimension as it changes from trial to trial.

The absence of a Stroop effect in the $R$ task is diagnostic for deciding the validity of our approach. The participants named cities in 774.6 and $781.5 \mathrm{msec}$, on average, for congruent and incongruent stimuli, respectively. The 6.9-msec difference was small and not significant statistically $\left[t(682)=0.34, M S_{\mathrm{e}}=20.50, p>.3\right]$. In Experiment 2 , too, the Stroop effect vanished for uncorrelated dimensions, in accord with the prediction of our contextual approach.

\section{Discussion}

The results of Experiment 1 were replicated in Experiment 2: The participants suffered Garner interference, yet only suffered Stroop interference when the irrelevant dimension was correlated with the relevant dimension. For the former, the participants did worse at naming a dimension if the other dimension varied orthogonally, as compared with a condition in which the latter was held constant. Country and city thus interacted in perception. We also found superior performance in the correlated dimensions tasks over that found in the random task. For the latter, in the $C$ tasks, defined by dimensional correlation, numerically large effects of Stroop emerged. In the $R$ task, by contrast, in which the dimensions were mutually uninformative, selective attention to the target succeeded, and as a result, no Stroop effect appeared. These results show again the incommensurability of dimensional correlation and selective attention.

\section{EXPERIMENT 3}

In both of the previous experiments, the participants were tested on the same set of countries and cities. That leaves open the possibility, however implausible, of an effect that could be attributed to the particular stimuli used. Therefore, in Experiment 3 we repeated the procedures of Experiments 1-2, with the following modifications. Each participant responded to a randomly different set of country-city compounds. We also introduced additional values of dimensional correlation. The aim of Experiment 3 was to replicate and extend our previous results.

\section{Method}

Participants. The participants were 13 Bar-Ilan University undergraduates, none of whom had performed in Experiments 1 and 2.

Stimuli and Apparatus. The stimuli and apparatus were the same as those of Experiment 1 , with two notable exceptions. First, a larger set of stimuli was included in the present experiment. We used 12 country names (Austria, Belgium, Brazil, Canada, China, England, France, Germany, India, Israel, Switzerland, and Uganda) and the respective 12 names of major cities within these countries (Salzburg, Antwerp, Rio de Janeiro, Toronto, Shanghai, Oxford, Marseilles, Munich, Bombay, Netanya, Geneva, and Entebbe) to create a set of 144 country-city compounds. On the basis of the results of our pilot study of 118 students, the 12 countries and 12 cities selected were ranked the most familiar. Of the pool of 12 country and 12 city names, 14 names ( 7 of each type) were drawn randomly, a different set for each participant. From the 49 countrycity pairs thus created, we constructed the same four experimental tasks $(R, C+, C-$, and $B)$ reflecting the manipulation of degree of dimensional correlation.

In Experiment 3, we also changed the proportion of congruent and incongruent stimuli within the $C+$ condition so that the probability of a congruent city-country combination was set to be four times higher than would have been expected on the basis of a strictly random sampling. As a result, $57 \%$ of the city-country combinations sampled were congruent, and $43 \%$ were incongruent (as compared with Experiments 1-2, in which $93 \%$ of the stimuli were congruent and $7 \%$ incongruent). We introduced the change to make the deviation from random sampling (i.e., zero dimensional correlation) symmetrical for positively and negatively correlated dimensions. Introducing another coefficient of dimensional correlation also extended the sample of values to test for effects on selective attention. In all other regards, the stimuli and apparatus were the same as those in Experiments 1 and 2. 
Procedure. The procedure was that of Experiments 1-2. On a trial, a pair of names appeared, flanking vertically the center of the screen, one of a country and another of a city. As in Experiment 1, country was the relevant dimension. The participants were encouraged to respond quickly without making errors.

\section{Results}

Average naming times are shown in panel $C$ of Figure 2. The participants responded faster in the $B$ task $(638.5 \mathrm{msec})$ than in the $R$ task $(711.4 \mathrm{msec})$, producing a significant Garner interference of $72.9 \mathrm{msec}[t(1099)=$ $\left.8.35, M S_{\mathrm{e}}=8.72, p<.001\right]$. The participants performed faster in the $C$ tasks than in the $R$ task by $32 \mathrm{msec}[C+$ task, $\left.679.39 \mathrm{msec} ; t(1140)=3.63, M S_{\mathrm{e}}=8.79, p<.001\right]$ and by $23.7 \mathrm{msec}[C-$ task, $687.64 \mathrm{msec} ; t(1175)=2.69$, $\left.M S_{\mathrm{e}}=8.80, p<.01\right]$. The $8.3-\mathrm{msec}$ difference between the $C+$ and the $C-$ tasks was not statistically significant $\left[t(1143)=-0.99, M S_{\mathrm{e}}=8.31, p>.2\right]$. Finally, Figure 2 also reveals that performance in the $B$ task was superior to performance in the $C+$ task by $40.9 \mathrm{msec}[t(1067)=5.05$, $\left.M S_{\mathrm{e}}=8.11, p<.001\right]$ and superior to that in the $C-$ task by $49.2 \mathrm{msec}\left[t(1102)=6.00, M S_{\mathrm{e}}=8.19, p<.001\right]$. An overall ANOVA confirmed the reliability of the differences in performance obtained across the various tasks $\left[F(3,2242)=24.59, M S_{\mathrm{e}}=3.05, p<.001\right]$. Clearly, the dimensions of country and city interacted in perception.

Regarding Stroop congruity, the pattern of results mimicked those of Experiments 1-2. Stroop effects emerged in the correlated tasks. In the $C+$ task, the participants responded faster to congruent country-city compounds (668.1 msec) than they did to incongruent compounds $(691.3 \mathrm{msec})$, producing a significant Stroop effect of $23.16 \mathrm{msec}\left[t(553)=1.99, M S_{\mathrm{e}}=11.65, p<.05\right]$. In the $C-$ task, the respective means were 668.7 and $688.5 \mathrm{msec}$ for congruent and incongruent pairs, producing a Stroop effect of $20 \mathrm{msec}$ that was not statistically significant $\left[t(588)=0.69, M S_{\mathrm{e}}=28.75, p=.2\right]$. Nevertheless, only 4 of the 13 participants failed to evince Stroop congruity in the $C$ - task. Under the hypothesis of an absence of the Stroop effect, the probability for such data equals $1 \%$. We concluded that fairly pervasive effects of Stroop congruity characterized the performance of the participants in the $C$ tasks.

Consider, finally, the $R$ task. The participants responded to congruent country-city compounds in an average of $704.4 \mathrm{msec}$ and responded to incongruent compounds in an average of $712.4 \mathrm{msec}$. The 8 -msec difference was not significant statistically $\left[t(585)=0.41, M S_{\mathrm{e}}=19.48, p>\right.$ .3]. Once again, when one dimension was not informative about the other, selective attention to the target was good. Consequently, the performance in the $R$ task was free of Stroop interference.

\section{Discussion}

The participants were unable to attend well to country in the face of irrelevant variation in city (Garner interference). In addition, the participants responded faster to stimuli entailing mostly associated country-city components (correlated tasks) than to stimuli entailing random combinations of city and country (random task; redundancy gain). Finally, congruent country-city compounds were named faster than incongruent ones within correlated contexts (Stroop effect), but not in random contexts (the absence of a Stroop effect). Taken together, these results indicate that processing of one dimension depended on the simultaneous processing of the other dimension.

In Experiments 1-3, we employed the same task: The participants had to name one dimension while ignoring another dimension as the values changed from trial to trial. Pooling the data, we first standardized them by subtracting the average RT value of each experiment from the raw data of that experiment and then performed a combined analysis on the difference scores.

For the pooled data of Experiments 1-3, we found a significant Garner interference of $59.7 \mathrm{msec}$ [ $t(3901)=$ $\left.12.25, M S_{\mathrm{e}}=4.87, p<.001\right]$. Performance in the $C+$ task was faster by $29.7 \mathrm{msec}$ than that in the $R$ task $[t(3985)=$ $\left.6.11, M S_{\mathrm{e}}=4.85, p<.001\right]$; similarly, performance in the $C$ - task was faster by $23.3 \mathrm{msec}$ than that in the $R$ task $\left[t(3980)=4.64, M S_{\mathrm{e}}=5.03, p<.001\right]$. The $6.3-\mathrm{msec}$ difference in performance between the $C+$ and the $C-$ tasks was not statistically significant $[t(3961)=-1.35$, $\left.M S_{\mathrm{e}}=4.69, p>.08\right]$. Finally, performance in the $B$ task was superior to performance in the $C+$ task by $30 \mathrm{msec}$ $\left[t(3882)=6.67, M S_{\mathrm{e}}=4.50, p<.001\right]$ and superior to that in the $C-$ task by $36.4 \mathrm{msec}\left[t(3877)=7.73, M S_{\mathrm{e}}=\right.$ $4.70, p<.001]$. An overall ANOVA performed on the entire data of Experiments 1-3 confirmed the reliability of the differences in performance obtained across the $R$, $C+, C-$, and $B$ tasks $\left[F(3,7862)=52.30, M S_{\mathrm{e}}=3.66\right.$, $p<.001]$.

For Stroop congruity, in the $C+$ tasks participants responded faster by $19.6 \mathrm{msec}$ to congruent than to incongruent stimuli, and the Stroop effect was significant $\left[t(1982)=2.29, M S_{\mathrm{e}}=8.53, p<.05\right]$. The participants responded faster to congruent than to incongruent pairs in the $C-$ tasks, too, producing a Stroop effect of $23.8 \mathrm{msec}$ $\left[t(1977)=1.37, M S_{\mathrm{e}}=17.30, p=.08\right]$. Of primary concern was the Stroop congruity observed in the $R$ tasks. Notably, no Stroop effect obtained in the $R$ tasks; the difference in RTs between congruent and incongruent compounds amounted to a minuscule $5.7 \mathrm{msec}[t(2001)=0.54$, $\left.M S_{\mathrm{e}}=10.68, p>.3\right]$. On the basis of the combined data of the three experiments, we conclude that dimensional correlation is a critical factor engendering the Stroop effect.

\section{EXPERIMENT 4}

In Experiments 1-3, the participants named the relevant dimension of word-word compounds, and dimensional correlation affected Stroop interference. In Experiments $4-5$, by contrast, the participants categorized the picture or the word of picture-word compounds. Naming may entail semantic processing, but more often it can be accomplished by the alternative routes of articulatory, phonetic, or graphemic processing (e.g., Glaser, 1992; Glaser \& Glaser, 1989; Smith \& Magee, 1980). Categorization, 
in contrast, mandates the extraction of meaning from the pertinent stimuli, because the very construct of a category is semantically established. Negatively stated, no categorization is possible in the absence of semantic processing.

Replacing the homogeneous word-word compounds with the heterogeneous picture-word compounds also entailed the introduction of a difference in relative dimensional discriminability. Categorization of pictures is uniformly faster than the categorization of words (Pellegrino, Rosinski, Chiesi, \& Siegel, 1977), because pictures engage the semantic system directly, whereas words first activate their nodes in the lexicon (Glaser, 1992). Consequently, incongruent pictures hamper the categorization of words, but incongruent words do not affect an already superior categorization performance with pictures (Smith \& Magee, 1980). The upshot is clear: For categorization, a semantic task through and through, pictures and words differ reliably in difficulty, with the more discriminable dimension (picture) bound to disrupt attention to the less discriminable dimension (word).

How does correlation affect attention to dimensions that differ appreciably in discriminability owing to qualitatively disparate routes of processing? Discriminability has been shown to be a major determinant of selectivity and Stroop effects. Can dimensional correlation alter the pattern of interference established by mismatched discriminability? For naming of word-word compounds (Experiments 1-3), the effect of dimensional correlation was amply registered. This experiment extended the scope of inquiry to categorization of picture-word compounds.

\section{Method}

Participants. The participants were 16 Bar-Ilan University undergraduates, none of whom had performed in the previous experiments.

Stimuli and Apparatus. We used words embedded within pictures as our stimuli. Out of the Snodgrass and Vanderwart (1980) standardized norms, we selected nine categories with eight items per category. The following categories and items were selected: $a n-$ imals (cat, cow, deer, dog, elephant, fox, gorilla, and mouse) kitchen utensils (bowl, cup, fork, frying pan, glass, kettle, knife, and pot), furniture (bed, chair, couch, dresser, lamp, rocking chair, stool, and table), body parts (arm, ear, eye, finger, hand, leg, lips, and nose), fruit (apple, banana, cherry, grapes, lemon, orange, peach, and pear), clothes (coat, dress, hat, pants, shirt, shoe, skirt, and tie), musical instruments (accordion, drum, flute, guitar, harp, piano, trumpet, and violin), vehicles (airplane, bicycle, bus, car, helicopter, motorcycle, train, and truck), and vegetables (carrot, corn, lettuce, mushroom, onion, pepper, potato, and tomato). A congruent stimulus comprised a picture and a word belonging in the same semantic category (e.g., the word mouse embedded within the picture of a dog), whereas an incongruent stimulus comprised a picture and a word belonging in separate semantic categories (e.g., the word apple embedded within the picture of a dog). We did not include compounds in which the picture and the word represented the same object. Also, a given item appeared either as a picture or as a word but did not appear in both forms for a given participant.

We used stratified random sampling of categories and items for each participant so that each participant was presented with a different set of stimuli. For category, seven of the nine categories were randomly chosen. For item, two of the eight items in each chosen category were randomly selected. Of the latter, one appeared in pic- ture form, the other as a word. A unique set of seven pictures and seven words resulted. Their combination yielded a $7 \times 7$ matrix of picture-word stimuli.

The stimuli were generated by an IBM-compatible (PC-486) $\mathrm{mi}$ crocomputer and displayed on a super-VGA 14-in. color monitor. The words were generated in Microsoft Word (Hebrew font Miriam, size 24). The pictures were line drawings adapted from the Snodgrass and Vanderwart (1980) pictures norms, digitized by a black-and-white image scanner (HP Scan Jet 5). A picture-word compound consisted of a word superimposed at the center of a picture. Viewed from a distance of approximately $60 \mathrm{~cm}$, the words subtended $0.57^{\circ}$ of visual angle in height and between $1.33^{\circ}$ and $5.16^{\circ}$ of visual angle in length. Pictures subtended between $2.38^{\circ}$ and $4.76^{\circ}$ of visual angle in height and between $5.71^{\circ}$ and $9.46^{\circ}$ of visual angle in length. On a trial, a single picture-word compound appeared on the screen. The stimuli appeared black over a light gray background at around the center of the screen. To avoid adaptation, we introduced a trial-to-trial spatial uncertainty of up to 10 pixels around the target locations.

From the 7 (picture) $\times 7$ (word) matrix, we constructed the same four experimental tasks $(R, C+, C-$, and $B)$ employed in Experiments 1-3. Our goal was to manipulate the degree of dimensional correlation in semantic category. To effect the changes in correlation, in each task we introduced a different value for the probability of a picture $B$ from category $m$ given a word $A$ from category $n$. The conditional probability, $p\left(B_{m} \mid A_{n}\right)$, within each of the four experimental tasks was the same as that in Experiment 3.

Procedure. The participants were tested individually in a dimly lit room. Each participant performed in each of the four tasks, with task order varying in a random fashion from participant to participant. On a trial, a picture-word compound appeared at the center of the screen. We introduced positional uncertainty so that there was a small trial-to-trial variation in the position of the compound. The relevant dimension in this experiment was the picture. Therefore, in each task, the participants categorized the pictures and tried to ignore the words. The participants were encouraged to respond quickly without making errors. Responses were made orally and recorded by a microphone. Each stimulus remained on display for $500 \mathrm{msec}$ or was response terminated, whichever came first. We measured the time elapsed between the onset of stimulus presentation and the vocal response in milliseconds. The experimenter recorded the correctness of the response.

We used the following procedure to familiarize the participants with the categories to appear in the experiment. First, the names of the seven categories selected for that participant appeared on the screen (centered, Hebrew font Miriam, size 24). The participants were instructed to memorize the seven category names and recite them out loud. The task was repeated to reach the criterion of $300 \%$ overlearning.

The experiment began with screen instructions. The participants were informed that, on a trial, they would see a picture-word compound--that is, a word embedded within a picture. They were further instructed to attend to the picture (and ignore the word) and categorize it according to the seven categories just learned. Following the instructions for a task, each participant completed nine practice trials (which mirrored the particular correlational structure used but were not analyzed); each task was followed by a short break. The participant initiated instructions to the next task by pressing the left key of the mouse. The experiment lasted approximately $15 \mathrm{~min}$.

\section{Results}

The mean RTs needed to categorize the pictures in the four experimental tasks are shown in panel D of Figure 2. The means differed across tasks, following the pattern expected on the basis of the results of Experiments 1-3. 
It took the participants longer to generate the category membership of a picture if the to-be-ignored word varied randomly from trial to trial $(824.2 \mathrm{msec})$ than if the word remained invariant $(771.9 \mathrm{msec})$. The difference in performance between the two tasks ( $R$ and $B$, respectively) amounted to a Garner interference of $52.3 \mathrm{msec}$ that was highly significant $\left[t(1331)=5.02, M S_{\mathrm{e}}=10.42, p<\right.$ $.001]$. Our participants noticed trial-to-trial changes in the irrelevant words when they generated the category of the picture.

If a correlation was introduced between the category of the picture and the category of the word, categorization performance with the pictures improved. Mean categorization time in the $C+$ task $(800.4 \mathrm{msec})$ was faster by $23.8 \mathrm{msec}$ than that in the $R$ task $\left[t(1353)=2.37, M S_{\mathrm{e}}=\right.$ $10.04, p<.01]$, and mean categorization time in the $C$ task ( $794.5 \mathrm{msec}$ ) was faster by $29.7 \mathrm{msec}$ than that in the $R$ task $\left[t(1324)=2.89, M S_{\mathrm{e}}=10.25, p<.01\right]$. The 5.9msec difference in performance between the $C+$ and the $C$ - tasks was not statistically significant $[t(1297)=0.58$, $\left.M S_{\mathrm{e}}=10.25, p>.2\right]$. These results imply that our participants were sensitive to the built-in correlation in semantic category between the word and the picture. When the respective categories covaried over trials, whether positively or negatively, performance was superior, as compared with that obtained in a task entailing no such correlation.

The gains observed in the $C$ tasks are notable for demonstrating that it was the covariation in category that the participants captured over the course of the experimental trials. Because all of the picture-word compounds presented were incongruent from the point of vantage of naming - none had the word naming the picture - the correlation established inhered in the semantic relation between categories of the pictures and the words. The participants perforce perceived the trial-to-trial variation in category of the irrelevant words (not merely the variation in their identity). Capitalizing on the covariation of category, the participants improved their performance (even in the negative case, in which the categories of the picture and the word never coincided).

Performance in the $B$ task was better than that in the $C$ tasks. Picture categorization in the $B$ task was faster by $28.6 \mathrm{msec}$, on average, as compared with that in the $C+$ task $\left[t(1304)=2.74, M S_{\mathrm{e}}=10.43, p<.01\right]$ and by $22.7 \mathrm{msec}$, on average, as compared with that in the $C$ task $\left[t(1275)=2.13, M S_{\mathrm{e}}=10.65, p<.05\right]$. An overall ANOVA confirmed the reliability of the previous differences $\left[F(3,2628)=8.75, M S_{\mathrm{e}}=3.67, p<.001\right]$.

Shown also in panel D of Figure 2 are the results pertaining to Stroop congruity. Consider, first, the performance in the $R$ task. The participants categorized pictures belonging in the same semantic category as the (to-be-ignored) words in an average of $824.2 \mathrm{msec}$, and they categorized pictures belonging in a different semantic category than the words in an average of $824.5 \mathrm{msec}$. The numerical parity indicates the absence of a Stroop effect in the $R$ condition. In the $C+$ task, too, the participants categorized pictures in category-congruent compounds in an average of $797.1 \mathrm{msec}$, and they categorized pictures in category-incongruent compounds in an average of $805.4 \mathrm{msec}$. The $8.3-\mathrm{msec}$ difference was not statistically significant $\left[t(662)=0.57, M S_{\mathrm{e}}=14.47, p>.2\right]$. In the $C$ - task, the participants categorized categorycongruent compounds in an average of $771.9 \mathrm{msec}$, but they categorized category-incongruent compounds an average of $795 \mathrm{msec}$. Although numerically sizable, the 23.2msec difference was not significant statistically $[t(633)=$ $0.46, M S_{\mathrm{e}}=50.46, p>.3 \mathrm{]}$. Hence, the correlations established between the categories of the pictures and those of the words did not generate significant Stroop effects in the present case. Although performance was somewhat facilitated with category-congruent stimuli, the facilitation was meager. The advantage of pictures over words in semantic processing yielded good selective attention to picture(-category) throughout the different tasks. The present imbalance across dimensions may also signal the boundary conditions for the power of dimensional correlation to exert its influence on performance.

\section{Discussion}

The two major findings of Experiment 4 were a substantial Garner interference across the $B$ and $R$ tasks but an absence of a Stroop effect within the $R$ and $C$ tasks. The Garner interference obtained implies that the participants did not selectively attend to the category of the picture and noticed variation in the irrelevant word. This conclusion is supported by the substantial amounts of performance gains obtained in the $C$ tasks. Pictures were categorized faster when the to-be-ignored words mostly shared their semantic category $(C+$ task $)$ or almost never shared their semantic category $(C-$ task) than when the categories were unrelated (the $R$ task). These results, duplicating the pattern observed in Experiments 1-3, demonstrate the effects of dimensional correlation on categorization of picture-word stimuli.

By our contextual approach, mismatched discriminability of the constituent dimensions is a major determinant of the Stroop effect. The different processes that pictures and words undergo during categorization (Glaser \& Glaser, 1989) likely result in a mismatched salience of the dimensions. The more discriminable dimension (picture) should not suffer intrusions from the less discriminable dimension (word). The outcome of Experiment 4 is commensurate with this prediction: Categorization of pictures was not plagued by Stroop interference even when the semantic categories of the components were correlated. Hence, correlation did not compromise the pattern of attentional outcome dictated by discriminability. By that pattern, the less discriminable dimension of word should be vulnerable to interference from the pictures. This prediction was tested in Experiment 5.

\section{EXPERIMENT 5}

\section{Method}

Participants. The participants were 22 Bar-llan University undergraduates, none of whom had performed in the previous experiments. 
Stimuli, Apparatus, and Procedure. The stimuli, apparatus, and procedure were identical to those in Experiment 4. However, in Experiment 5, the participants categorized the words and had to ignore the pictures within which the words were embedded

\section{Results}

Shown in panel E of Figure 2 are the average RTs needed to categorize words in the four experimental tasks. The participants generated the category of the word more rapidly when the picture was invariant $(877.6 \mathrm{msec})$ than when the picture varied randomly from trial to trial $(965.4 \mathrm{msec})$. The difference, $87.9 \mathrm{msec}$, amounted to a large Garner interference that was highly significant $\left[t(1888)=8.73, M S_{\mathrm{e}}=10.06, p<.001\right]$. Our participants could not ignore variation along the irrelevant dimension of picture while categorizing the words.

Categorization of the words was faster in tasks in which there was a correlation between the categories of the pictures and those of the words than in a task in which there was zero dimensional correlation. Performance in the $C+$ task $(941.2 \mathrm{msec})$ was faster by $24.2 \mathrm{msec}$ than that in the $R$ task $\left[t(1854)=2.26, M S_{\mathrm{e}}=10.69, p<.05\right]$, and categorization performance in the $C$ - task $(923.8 \mathrm{msec})$ was faster by $41.6 \mathrm{msec}$ than that in the $R$ task [ $t(1863)=$ $\left.3.82, M S_{\mathrm{e}}=10.87, p<.001\right]$. The 17.4-msec difference between the $\mathrm{C}+$ and the $C$ - tasks was just not significant statistically $\left[t(1839)=1.59, M S_{\mathrm{e}}=10.90, p>.06\right]$. These findings indicate that the participants captured the correlation between the semantic category of the picture and the semantic category of the word to improve their performance.

A glance at panel $\mathrm{E}$ of Figure 2 also reveals that performance in the $B$ task was superior to that in the $C+$ task by $63.6 \mathrm{msec}\left[t(1864)=6.32, M S_{\mathrm{e}}=10.07, p<.001\right]$ and was superior to that in the $C$ - task by $46.3 \mathrm{msec}[t(1873)=$ $\left.4.50, M S_{\mathrm{e}}=10.27, p<.001\right]$. The redundancy gain entailed in the correlation between dimensions could not override the cost incurred to performance by the spatial separation of components. The results of an overall ANOVA agreed with those of the previous analyses regarding the differences across the tasks $[F(3,3727)=$ $\left.25.39, M S_{\mathrm{e}}=3.74, p<.001\right]$

Of central interest in the Stroop analyses was the difference in performance between category-congruent and category-incongruent stimuli in the $R$ task (see the empty columns in panel $\mathrm{E}$ of Figure 2). Generating the category of the word was faster when it was drawn from the semantic category of the irrelevant picture $(924.5 \mathrm{msec})$ than when category membership conflicted $(972.3 \mathrm{msec})$. The 47.8-msec difference in performance amounted to a significant Stroop effect $\left[t(938)=2.23, M S_{\mathrm{e}}=21.39\right.$, $p<.05]$. Hence, despite the zero correlation, selective attention failed to the category of the relevant word.

For the correlated tasks, too, the words in categorycongruent compounds were categorized faster than those in category-incongruent ones. In the $C+$ task, the participants responded $23.6 \mathrm{msec}$ faster to congruent stimuli (931.24 msec) than to incongruent stimuli [954.9 msec; $\left.t(914)=1.54, M S_{\mathrm{e}}=15.32, p=.06\right]$. In the $C-$ task, the participants categorized congruent compounds $62.7 \mathrm{msec}$ faster than they categorized incongruent ones [863.5 vs. $\left.926.2 \mathrm{msec} ; t(923)=1.53, M S_{\mathrm{e}}=41.05, p=.06\right]$. Hence, under the correlated conditions, selective attention was plagued by Stroop effects that were as sizable as that observed under the zero correlation condition.

Because categorization requires semantic analysis-a task in which pictures are engaged ahead of words --performance with words is bound to suffer interference from the pictures. By Glaser and Glaser's (1989) account, the Stroop effects that plagued word categorization in all the conditions were inevitable and are not indeed surprising. Our contextual approach complements this account: The dimensional mismatch favoring pictures disrupted categorization performance with words-the less salient dimension. Supporting this contention is the large difference in speed observed between categorization of pictures (Experiment 4) and that of words (Experiment 5) of the same picture-word compounds. Although different persons participated in the two experiments, the 128 -msec advantage for pictures is suggestive (means of 798 and $926 \mathrm{msec}$, respectively, for categorization in Experiments 4 and 5).

Nevertheless, dimensional correlation did affect performance even in Experiment 5. The superior performance in the $C$ tasks, as compared with that in the $R$ task, is attributable to the correlation of categories. The participants were sensitive to the built-in covariation of category and used that covariation to improve performance.

\section{Discussion}

The Garner interference observed in categorization of words registers the toll exacted by trial-to-trial variation of the irrelevant picture. The superior performance observed when the category of the picture and that of the word covaried betrays the fact that, under the present dimensional mismatch, correlation still exerted its influence. The ubiquitous Stroop effect in Experiment 5 (in the correlated tasks, as well as in the zero correlation task) is easily accounted for by the Glaser and Glaser (1989) model: Because pictures, but not words, have privileged access to the semantic information producing the category, the words suffered intrusions from the irrelevant pictures. Once noticed, picture category was registered as either congruent or conflicting with that of the word. The difference in performance between the two types of correspondence produced the Stroop effect. By Melara and Algom's account (1996), the failure to satisfy the stipulation of matched discriminability resulted in the emergence of a Stroop effect. For categorization of words -less salient than pictures-a Stroop effect emerged even under conditions of random allocation of dimensional values.

Taken together, the results of Experiments 4-5 tap the interplay between the contextual variables of discriminability and correlation in determining the outcome of the Stroop experiment. They demonstrate that the dimensional imbalance between picture and word suffices to engender substantial Stroop effects with the less efficient process. The mismatch in discriminability also marks 
the boundary conditions for the effects of correlation to exert themselves. When the dimensional mismatch is large, Stroop effects will plague performance with the less salient dimension regardless of the correlation between the dimensions (Algom et al., 1996). Under such conditions, dimensional correlation weakly modulates the magnitude of an inevitable Stroop effect.

\section{GENERAL DISCUSSION}

The collective results of Experiments 1-3 show that dimensional correlation had a profound effect on selective attention. When it was zero--a condition in which neither component of the stimulus carried information about the other component--selective attention to the target was good (when gauged by Stroop interference, although not when gauged by Garner interference). On the other hand, when dimensional values covaried systematically, whether positively or negatively, substantial Stroop and Garner effects plagued performance on the target dimension. The collective results of Experiments 4-5 demonstrated the influence on selective attention of an additional variable of context--dimensional discriminability. Picture-word stimuli engendered qualitatively different processing of their constituent dimensions, a disparity that was transformed into a corresponding mismatch in discriminability favoring pictures in categorization. In Experiments $4-5$, pictures interfered with the categorization of words, but irrelevant words did not interfere with the categorization of pictures. The mismatch in discriminability undermined the potency of dimensional correlation to govern the attentional outcome. Nevertheless, correlation still modulated categorization performance even when the pattern of interference was largely determined by relative dimensional discriminability.

\section{A Contextual Account of the Stroop Effect}

Our data, taken as a whole, underscore the power of contextual variables - notably, of dimensional correlation - to determine the outcome of the Stroop experiment. Given these results, it is puzzling to recognize that investigators have recurrently imputed correlations into their designs, thereby adjusting the outcome in advance. The first investigator to introduce a correlation between the values of the constituent dimensions was Stroop himself, who used only incongruent stimuli in his study. Modern investigators include congruent stimuli and incongruent stimuli in equal numbers (but see Logan \& Zbrodoff, 1979, 1998 , for notable exceptions). Here is the makeup of the typical Stroop experiment. Four color words and their corresponding colors are used. Each color word appears printed in its matching color nine times, making for a set of 36 congruent trials. Each color word also appears printed in each of the other three colors three times, making for another set of 36 incongruent trials (frequently, a control set of 36 neutral words is also used). Note the disparity in probability of presentation: A word appears printed in its corresponding color three times as often as it does in each of the other colors! Therefore, in this most common of designs, the word is predictive of color (and vice versa), and the correlation is bound to undermine selective attention. One does not need to invoke the notions of automaticity, speed of processing, or indeed, any notion related to semantic analysis, to explain the Stroop effect obtained. The correlation imputed has already determined the presence of the Stroop effect, the magnitude of the effect, and the direction of the effect (whether Stroop or reverse Stroop). These startling results and conclusions are best understood within the framework of our contextual approach to the Stroop phenomenon (cf. Melara \& Algom, 1996; see Pomerantz, 1991, for further strategic influences on the success or failure of selective attention).

A basic tenet of our contextual approach is this. The more salient and informative a distractor is about the target-expressed, respectively, by relative dimensional discriminability and dimensional correlation - the more likely it is that selective attention to the target will fail. For discriminability, the makeup or complexity of the stimulus attributes may engender an imbalance favoring the word dimension: The trial-to-trial changes in words are experienced as being psychologically greater than the trial-to-trial changes in colors. This form of dimensional imbalance is typical for most previous research with Stroop stimuli. The greater salience of words necessarily undermines selective attention to the colors. Theoretically, however, neither the imbalance nor its form is fixed or inevitable. A different set of experimental stimuli (e.g., different fonts of the word and/or different print colors) will give rise to a different unitization of values in the $\operatorname{cog}$ nitive space.

For dimensional correlation, the effect on selectivity is determined by the way an experimenter allocates the values of color and word to form the experimental stimuli. This allocation - indeed, the experimental design - fixes the conditional probability of the word, given the color, and the conditional probability of the color, given the word. Most Stroop researchers have shunned the truly random design of value allocation, electing instead to equate the number of congruent and incongruent stimuli in the set. In this way, the investigators have built a predictive relationship between words and colors into the design. Observers notice the predictive relationship, the information thus provided jeopardizing selective attention.

On the basis of other manipulations of context, Besner and his associates (e.g., Bauer \& Besner, 1997; Besner \& Stolz, 1999; Besner, Stolz, \& Boutilier, 1997; Stolz \& Besner, 1999) independently reached conclusions similar to the present ones. They concluded that "the Stroop effect is reduced in magnitude or eliminated, depending on details of context. Contrary to the widespread view that it reflects automatic processing, the Stroop effect is better conceptualized as reflecting the action of . . attentional systems" (Besner \& Stolz, 1999, p. 99) and that, "in contrast to the standard claim that words automatically activate their meanings, our view is that semantic-level activation is subject to attentional control" (Stolz \& Besner, 1999, p. 64). Pomerantz's (1991) keen observations on the 
dependence of selectivity on strategic control by the perceiver are also inhospitable to views of strong automaticity. Our results and conclusions are commensurate with those of Besner and Pomerantz. In our view, the Stroop task is a microcosm of everyday attentional processing, exemplifying the inherent incompatibility between the drive to seek information and the goal of ignoring it. The main point to note is that the experimenter, by creating his or her stimuli and by conceiving the design, indirectly governs attentional processing and influences the outcome.

\section{REFERENCES}

Algom, D., Dekel, A., \& PANSKY, A. (1993). On the perception of number. In A. Garriga-Trillo, P. R. Minon, C. Garcia-Gallego, C. Lubin, J. M. Merino, \& A. Villarino (Eds.), Fechner Day '93 (pp. 1-6). Palma de Mallorca: International Society for Psychophysics.

Algom, D., Dekel, A., \& Pansky, A. (1996). The perception of number from the separability of the stimulus: The Stroop effect revisited. Memory \& Cognition, 24, 557-572.

Alloy, L. B., \& TABACHNIK, N. (1984). Assessment of covariation by humans and animals: The joint influence of prior expectations and current situational information. Psychological Review, 91, 112-149.

ARIEH, A., \& ALGOM, D. (1997). Reading words in Stroop-like tasks: The effect of contingency between semantic and visual components. Psychologia, 6, 7-19. (Hebrew)

Bar-Hillel, M., \& Wagenaar, W. A. (1991). The perception of randomness. Advances in Applied Mathematics, 12, 428-454.

Bauer, B., \& Besner, D. (1997). Processing in the Stroop task: Mental set as a determinant of performance. Canadian Journal of Experimental Psychology, 51, 61-68.

BESNER, D., \& STOLZ, J. A. (1999). What kind of attention modulates the Stroop effect? Psychonomic Bulletin \& Review, 6, 99-104.

Besner, D., Stolz, J. A., \& Boutilier, C. (1997). The Stroop effect and the myth of automaticity. Psychonomic Bulletin \& Review, 4, 221-225

Dunbar, K. N., \& MaCLeod, C. M. (1984). A horse race of a different color: Stroop interference patterns with transformed words. Journal of Experimental Psychology: Human Perception \& Performance, 10, 622-639.

GARNER, W. R. (1962). Uncertainty and structure as psychological concepts. New York: Wiley

GARNER, W. R. (1974). The processing of information and structure. Potomac, MD: Erlbaum.

GaRner, W. R., \& Felfol.DY, G. L. (1970). Integrality of stimulus dimensions in various types of information processing. Cognitive Psychology, 1, 225-241.

Glaser, W. R. (1992). Picture naming. Cognition, 42, 61-105.

Glaser, W. R., \& Glaser, M. O. (1989). Context effects in Stroop-like word and picture processing. Journal of Experimental Psychology: General, 118, 13-42.

HASHER, L., \& ZaCKS, R. T. (1979). Automatic and effortful processes in memory. Journal of Experimental Psychology: General, 108, 356388.

KahNeman, D., \& ChaJCZYK, D. (1983). Tests of automaticity of reading: Dilution of Stroop effects by color-irrelevant stimuli. Journal of Experimental Psychology: Human Perception \& Performance, 9 , 497-509.

Kahneman, D., \& Treisman, A. (1984). Changing views of attention and automaticity. In R. Parasuraman \& D. R. Davies (Eds.), Varieties of attention (pp. 29-61). Orlando, FL: Academic Press.

KaReEv, Y. (1995). Positive bias in the perception of covariation. Psychological Review, 102, 490-502.

Kareev, Y., Lieberman, I., \& Lev, M. (1997). Through a narrow window: Sample size and the perception of correlation. Journal of Experimental Psychology: General, 126, 278-287.

LoGan, G. D. (1980). Attention and automaticity in Stroop and priming tasks: Theory and data. Cognitive Psychology, 12, 523-553.

LOGAN, G. D. \& ZBRODOFF, N. J. (1979). When it helps to be misled:
Facilitative effects of increasing the frequency of conflicting stimuli in a Stroop-like task. Memory \& Cognition, 7, 166-174.

Logan, G. D., \& ZBrodofF, N. J. (1998). Stroop-type interference: Congruity effects in color naming with typewritten responses. Journal of Experimental Psychology: Human Perception \& Performance, 24, 978-992

MACLEOD, C. M. (1991). Half a century of research on the Stroop effect: An integrative review. Psychological Bulletin, 109, 163-203.

MacLeod, C. M. (1992). The Stroop task: The "gold standard" of attentional measures. Journal of Experimental Psychology: General, $121,12-14$.

MACLeOD, C. M., \& Hodder, S. L. (1998). Presenting two incongruent color words on a single trial does not alter Stroop interference. Memory \& Cognition, 26, 212-219.

Melara, R. D., \& Algom, D. (1996, October). Stroop and selective attention: Putting it all in context. Paper presented at the 37th Annual Meeting of the Psychonomic Society, Chicago.

Melara, R. D., \& Mounts, J. R. W. (1993). Selective attention to Stroop dimensions: Effects of baseline discriminability, response mode, and practice. Memory \& Cognition, 21, 627-645.

Melara, R. D., \& MOUNTS, J. R. W. (1994). Contextual influences on interactive processing: Effects of discriminability, quantity, and uncertainty. Perception \& Psychophysics, 56, 73-90.

PANSKY, A., \& AlgOM, D. (1999). Stroop and Garner effects in comparative judgment of numerals: The role of attention. Journal of Experimental Psychology: Human Perception \& Performance, 25, 38-58.

Pellegrino, J. W., Rosinski, R. R., ChIESI, H. L., \& Siegel, A. (1977). Picture-word differences in decision latency: An analysis of single and dual memory models. Memory \& Cognition, 5, 383-396.

Pomerantz, J. R. (1983). Global and local precedence: Selective attention in form and motion perception. Journal of Experimental Psychology: General, 112, 515-540.

Pomerantz, J. R. (1986). Visual form perception: An overview. In E. C. Schwab \& H. C. Nusbaum (Eds.), Pattern recognition by humans and machines: Visual perception (pp. 1-30). New York: Academic Press.

Pomerantz, J. R. (1991). The structure of visual configurations: Stimulus versus subject contributions. In G. R. Lockhead \& J. R. Pomerantz (Eds.), The perception of structure: Essays in honor of Wendel R. Garner (pp. 195-210). Washington, DC: American Psychological Association.

Pomerantz, J. R., Pristach, E. A., \& Carson, C. E. (1989). Attention and object perception. In B. E. Shepp \& S. Ballesteros (Eds.), Object perception: Structure and process (pp. 53-89). Hillsdale, NJ: Erlbaum.

POSNER, M. I. (1978). Chronometric explorations of the mind. Hillsdale, NJ: Erlbaum.

Sabri, M., Melara, R. D., \& Algom, D. (in press). A confluence of contexts: Asymmetric versus global failure of selective attention to Stroop dimensions. Journal of Experimental Psychology: Human Perception \& Performance.

Shalev, L., \& Algom, D. (2000). Stroop and Garner effects in and out of Posner's beam: Reconciling two conceptions of selective attention. Journal of Experimental Psychology: Human Perception \& Performance, 26, 997-1017.

SMith, M. C., \& MageE, L. E. (1980). Tracing the time course of picture-word processing. Journal of Experimental Psychology: General, 109, 373-392.

SNODGRASS, J. G., \& VANDERWART, M. (1980). A standardized set of 260 pictures: Norms for name agreement, image agreement, familiarity, and visual complexity. Journal of Experimental Psychology Human Learning \& Memory, 6, 174-215.

StOLZ, J. A., \& BESNER, D. (1999). On the myth of automatic semantic activation in reading. Current Directions in Psychological Science, 8 , 61-65.

StroOP, J. R. (1935). Studies of interference in serial verbal reactions. Journal of Experimental Psychology, 18, 643-662.

(Manuscript received February 15, 1999; revision accepted for publication April 24, 2000.) 